\title{
UTILIZAÇÃO DE RESÍDUO DA INDÚSTRIA CERVEJEIRA NA BIOSSORÇÃO DE ÍONS METÁLICOS PRESENTES EM ÁGUAS SUBTERRÂNEAS
}

\author{
I. B. FONTANA ${ }^{1}$, M. PETERSON ${ }^{1}$ e M. A. P. CECHINEL ${ }^{1}$ \\ ${ }^{1}$ Universidade do Extremo Sul Catarinense, Unidade Acadêmica de Ciências, Engenharias e \\ Tecnologias - UNACET, Departamento de Engenharia Química \\ E-mail para contato: maria.cechinel@unesc.net
}

\begin{abstract}
RESUMO - Amostras de águas subterrâneas foram coletadas e caracterizadas em análises físico-químicas, verificando-se a presença de íons $\mathrm{Fe}(\mathrm{II})$ e $\mathrm{Mn}$ (II) acima dos valores máximos permitidos (VMP) pela legislação brasileira para consumo humano. Foram realizados estudos de biossorção utilizando-se bagaço de malte, em batelada, variando-se a dosagem de biomassa. Ensaios cinéticos mostraram que o equilíbrio de biossorção é atingido em até $480 \mathrm{~min}$, com percentuais de remoção de aproximadamente $87 \%$ e $71 \%$ para $\mathrm{Fe}^{2+}$ e $\mathrm{Mn}^{2+}$, respectivamente. A capacidade máxima de biossorção para o $\mathrm{Fe}^{2+}$ e $\mathrm{Mn}^{2+}$ foi de $1,2 \mathrm{mg} / \mathrm{g}$ e $0,6 \mathrm{mg} / \mathrm{g}$, respectivamente. O modelo cinético de pseudo-segunda ordem apresentou melhor ajuste aos dados experimentais.
\end{abstract}

\section{INTRODUÇÃO}

A biossorção tem sido considerada uma alternativa promissora quando comparada a outras tecnologias tradicionais de tratamento de águas, uma vez que é de baixo custo e ambientalmente amigável, especialmente quando uma biomassa natural é utilizada (Ibrahim, 2011). Muitos materiais de origem biológica já foram investigados na remoção de poluentes de águas contaminadas. A aplicação de resíduos de biomassa microbiana e da indústria de alimentos como biossorventes vem ganhando destaque devido ao grande volume produzido (Wang e Chen, 2006) e por ser um material de baixo custo. Dentre esses materiais, destaca-se o bagaço de malte, subproduto da indústria cervejeira, que é composto pela casca do malte, fragmentos da camada de aleurona, plúmula, restos de parede celular e proteína coagulada. Atualmente, em função de seu alto teor de fibras, o resíduo do malte é destinado, majoritariamente, à nutrição animal (Aquarone et al., 2001). Como o setor cervejeiro no Brasil, principalmente o artesanal, vem crescendo significativamente nos últimos 10 anos, conferir um valor tecnológico a essa biomassa pode ser uma alternativa interessante, visto que há uma produção expressiva do resíduo, podendo chegar a $20 \mathrm{~kg}$ de bagaço de malte para cada $100 \mathrm{~L}$ de cerveja fabricada (Santos e Ribeiro, 2012).

A presença íons em águas subterrâneas, mesmo que em pequenas quantidades, afetam a utilidade da água para algumas finalidades domésticas e industriais. Além disso, por se tratar de uma região carbonífera, a presença de metais como $\mathrm{Fe}^{2+}$ e $\mathrm{Mn}^{2+}$ pode estar relacionada à diluição de águas oriundas da drenagem ácida de mina (DAM) nas fontes de água (Akcil e Koldas, 2006). 
O trabalho teve como objetivo utilizar o bagaço de malte como biossorvente na remoção de poluentes presentes em águas subterrâneas (íons $\mathrm{Fe}^{2+}$ e $\mathrm{Mn}^{2+}$ ), visando uma possível aplicação para o consumo animal e humano e o abastecimento industrial.

\section{MATERIAIS E MÉTODOS}

As amostras de águas subterrâneas foram coletadas em diversos pontos do município de Urussanga, localizado no sul do estado de Santa Catarina - Brasil, num total de 9 amostras. Os parâmetros de qualidade analisados para cada amostra $(\mathrm{pH}$, condutividade, sólidos dissolvidos, cor, turbidez, ferro e manganês) seguiram os procedimentos analíticos apresentados no APHA/AWWA/WEF (2012).

O bagaço de malte utilizado foi cedido por uma indústria cervejeira localizada no sul do estado de Santa Catarina - Brasil. O material foi lavado com água da torneira e água destilada para remover possíveis impurezas e, em seguida, foi seco ao sol e posteriormente levado a secagem em estufa a $70{ }^{\circ} \mathrm{C}$ por 24 horas para remoção do excesso de umidade. A área superficial e o volume total dos poros do biossorvente foram determinados a partir da isoterma de adsorção de $\mathrm{N}_{2}$. A estrutura superficial e a morfologia do biomaterial foram caracterizadas por Microscopia Eletrônica de Varredura (MEV). A análise qualitativa dos grupos funcionais presentes na superfície do bagaço de malte foi obtida por Espectroscopia no Infravermelho com Transformada de Fourier (FTIR).

Para o estudo de biossorção, preparou-se uma solução sintética contento aproximadamente $1,5 \mathrm{mg} / \mathrm{L}$ e $2,5 \mathrm{mg} / \mathrm{L}$ de íons $\mathrm{Mn}^{2+}$ e Fe ${ }^{2+}$, pela dissolução dos sais de $\mathrm{FeSO}_{4} \cdot 7 \mathrm{H}_{2} \mathrm{O}\left(99,0 \%\right.$, Synth) e $\mathrm{MnSO}_{4} \cdot \mathrm{H}_{2} \mathrm{O}(>98,0 \%$, Fmaia) em água destilada. Para avaliar a influência da concentração de biomassa na remoção dos íons metálicos, foram realizados ensaios em sistema batelada em frascos Erlenmeyer de $100 \mathrm{~mL}$, em que $50 \mathrm{~mL}$ da solução sintética foram colocados em contato com o bagaço de malte em razões de massa/volume variando de 0,5 a 4,0 g/L. Os ensaios cinéticos em sistema batelada também foram realizados em frascos Erlenmeyer de $100 \mathrm{~mL}$ com $50 \mathrm{~mL}$ da solução sintética e 2,0 g/L de bagaço de malte. A fim de investigar o mecanismo de biossorção, os modelos cinéticos de pseudoprimeira ordem e pseudo-segunda ordem foram ajustados aos dados experimentais (Lagergren, 1898).

\section{RESULTADOS E DISCUSSÕES}

\subsection{Caracterização físico-química das águas subterrâneas e da biomassa}

As amostras de águas subterrâneas coletadas apresentaram pH neutro $(6,6 \pm 0,8)$, elevada condutividade $(174 \pm 60 \mu \mathrm{S} / \mathrm{cm})$, baixa presença de sólidos dissolvidos totais $(87 \pm 50$ $\mathrm{mg} / \mathrm{L})$, cor $(8 \pm 5 \mathrm{uH})$ e turbidez $(12 \pm 8 \mathrm{uT})$ acimas dos VMP definidos pela legislação vigente (Brasil, 2011). Os íons Ferro e Manganês foram identificados em todas as amostras coletadas, apresentando valores médios de 0,8 $\pm 0,7$ e $0,35 \pm 0,06 \mathrm{mg} / \mathrm{L}$, respectivamente, que estão acima do VMP. Por se tratar de uma região carbonífera, a presença destes metais pode estar relacionada à diluição de águas oriundas da drenagem ácida de mina (DAM) nas fontes de água. 
Com relação à caracterização físico-química da biomassa, a área superficial e o volume total de poros do bagaço de malte apresentaram valores iguais a $0,171 \mathrm{~m}^{2} / \mathrm{g} \mathrm{e} 4,516 \times 10^{-4}$ $\mathrm{cm}^{3} / \mathrm{g}$, respectivamente. Os resultados mostram que há poucos poros no bagaço de malte, $\mathrm{o}$ que justifica a sua baixa área superficial e encontra correspondência com outros estudos realizados com bagaço de malte (Fontana et al., 2016). A Figura 1 apresenta as micrografias obtidas por MEV para ampliação de 160 e 499 vezes. Observa-se que o material apresenta uma estrutura rígida, com a superfície pouco irregular. Poros são observados apenas no corte transversal da biomassa (Figura 1b), onde é possível verificar canais regulares que provavelmente compõe o sistema vascular do vegetal.

Figura 1 - Micrografias do bagaço de malte com ampliação de (a) 160 e (b) 499 vezes.
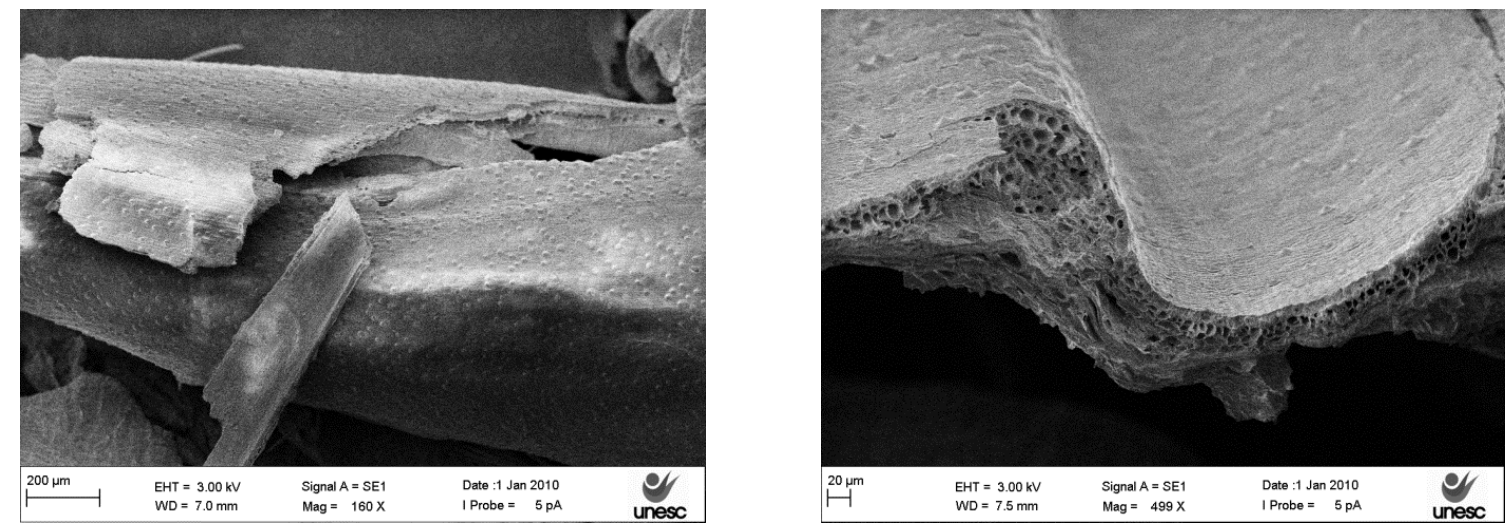

O espectro FTIR do bagaço de malte é ilustrado na Figura 2. Os picos característicos encontram-se a 2920 e $2850 \mathrm{~cm}^{-1}$, que correspondem a vibrações do estiramento $\mathrm{C}-\mathrm{H}$ em cadeias alifáticas $(-\mathrm{CH} 2 \mathrm{e}-\mathrm{CH} 3)$, que podem pertencer à celulose, lignina ou hemicelulose (Ferraz et al., 2015). Também é possível observar picos a 1740 e $1630 \mathrm{~cm}^{-1}$, sendo o primeiro característico da vibração do estiramento do grupo carbonilo $(\mathrm{C}=\mathrm{O})$ em cetonas, éteres, aldeídos e ácidos carboxílicos e o segundo atribuído ao grupo carbonilo em anéis aromáticos encontrados em lignina (Ferraz et al., 2015). Os grupamentos identificados no espectro estão de acordo com a composição do bagaço, que é rico em celulose (16-21\%), hemicelulose (1529\%), lignina (19-28\%) e proteínas (24-39\%) (Santos et al., 2015).

Figura 2 - Espectro FTIR do bagaço de malte.

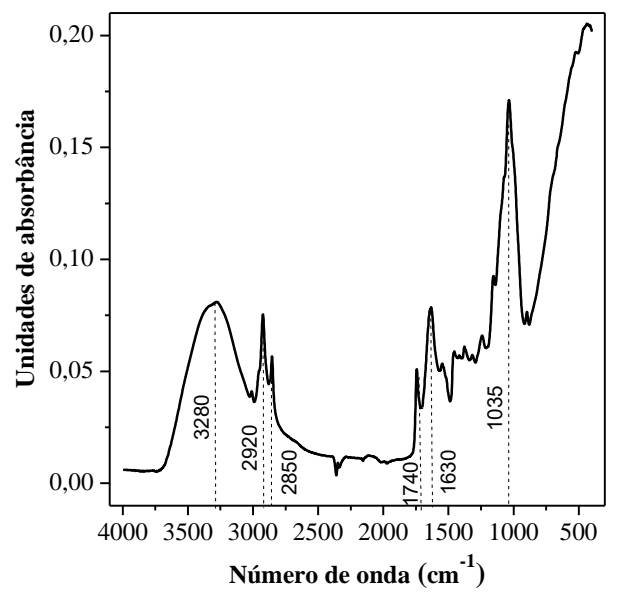




\subsection{Efeito da dosagem de biossorvente e cinética de biossorção}

A Figura 3 apresenta os resultados obtidos para a biossorção de Fe e Mn para diferentes quantidades de bagaço de malte. Observa-se a redução dos íons metálicos na fase líquida com o aumento da concentração de biomassa até uma razão de 2,0 g/L.

Figura 3 - Concentração dos íons metálicos em fase líquida para diferentes razões de biossorvente: $\square$ - Ferro; $\bigcirc$ - Manganês.

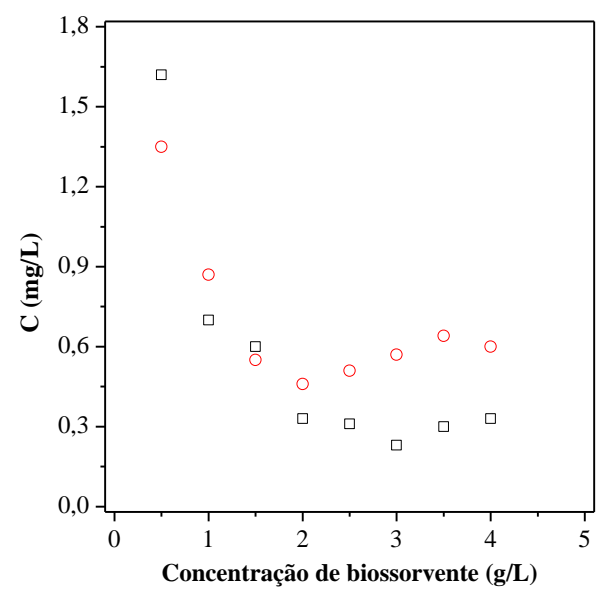

Este comportamento pode ser atribuído à maior área de superfície e à disponibilidade de mais sítios ativos para a adsorção. De acordo com Fadel et al. (2015), quando a concentração de biomassa é baixa, os íons metálicos na solução não só seriam adsorvidos na superfície da biomassa, como também entrariam na parte intracelular, facilitando o gradiente de concentração de íons metálicos. Para o íon $\mathrm{Mn}^{2+}$ observou-se um pequeno aumento na concentração em fase líquida após $2,0 \mathrm{~g} / \mathrm{L}$ de biossorvente, que pode estar associado a um aumento das interações eletrostáticas com alta concentração de biomassa, o que inibe a biossorção de metais.

Comparando-se os resultados obtidos para a concentração ótima de biomassa (2,0 g/L), observou-se uma remoção de $88 \%$ e $83 \%$ para Fe e Mn, com uma capacidade de remoção de $1,1 \mathrm{mg} / \mathrm{L}$ e $0,5 \mathrm{mg} / \mathrm{L}$, respectivamente. A capacidade de remoção de Fe corresponde a duas vezes a capacidade de remoção de $\mathrm{Mn}$, resultado que pode ser justificado pelo fato de a concentração inicial de Ferro ser maior que a de Manganês. Além disso, quando se tem cátions de mesma valência, a afinidade na adsorção é determinada, principalmente, pelo raio iônico, visto que quanto menor o raio iônico de um íon metálico, maior será a taxa de adsorção (Igwe e Abia, 2007). Comparando-se os raios iônicos dos íons metálicos utilizados $\mathrm{Fe}^{2+}(0,78 \AA)$ e $\mathrm{Mn}^{2+}(0,83 \AA)$, percebe-se que o resultado está de acordo com a teoria apresentada na literatura ( $\mathrm{Ji} \mathrm{e} \mathrm{Li}, 1997)$.

A Figura 4 apresenta o perfil cinético de concentração dos íons Fe e Mn na fase líquida e sólida. Observa-se que a biossorção ocorre rapidamente na fase inicial do experimento, diminuindo a concentração na fase líquida dos íons $\mathrm{Fe}^{2+}$ e $\mathrm{Mn}^{2+}$ em $79 \%$ e $59 \%$ nas primeiras $2 \mathrm{~h}$. Como a concentração inicial de íons metálicos é baixa, a proporção de poluentes em relação aos sítios ativados é pequena, de modo que os íons podem facilmente e 
rapidamente se ligar aos locais com maior energia. A Tabela 1 mostra os valores obtidos para as constantes cinéticas de adsorção para os modelos cinéticos de pseudo-primeira ordem e pseudo-segunda ordem. Os resultados obtidos para as constantes $\mathrm{q}_{1}$ não são significativos, visto que se afastaram dos valores obtidos para $\mathrm{q}_{\mathrm{exp}}$, tanto para o Fe quanto para o Mn. Desta forma, conclui-se que a biossorção não segue a cinética de pseudo-primeira ordem. Os valores de $\mathrm{q}_{2}$ calculados com o modelo cinético de pseudo-segunda ordem são muito próximos do valor experimental para os dois íons metálicos, apresentando também valores de $\mathrm{R}^{2}$ muito próximos de 1 . Desta forma, conclui-se que a adsorção de Fe e Mn pelo bagaço de malte obedece a cinética de pseudo-segunda ordem. Resultados semelhantes foram obtidos por Zhang et al., (2014), cujos dados experimentais ajustaram-se melhor ao modelo de pseudo-segunda ordem, com coeficiente de correlação linear $\mathrm{R}^{2}$ próximo a 1 , bem como valores de $\mathrm{q}_{2}$ de acordo com os valores de $\mathrm{q}_{\text {exp }}$, o que também confirma a validade desse modelo para a adsorção de Fe e Mn em bagaço de malte.

Figura 4 - Perfil cinético de concentração na fase líquida (símbolos vazados) e sólida (símbolos preenchidos) para os íons metálicos $\mathrm{Fe}^{2+}(\square$ e $\mathbf{\square})$ e $\mathrm{Mn}^{2+}(\bigcirc$ e $\bigcirc)$.

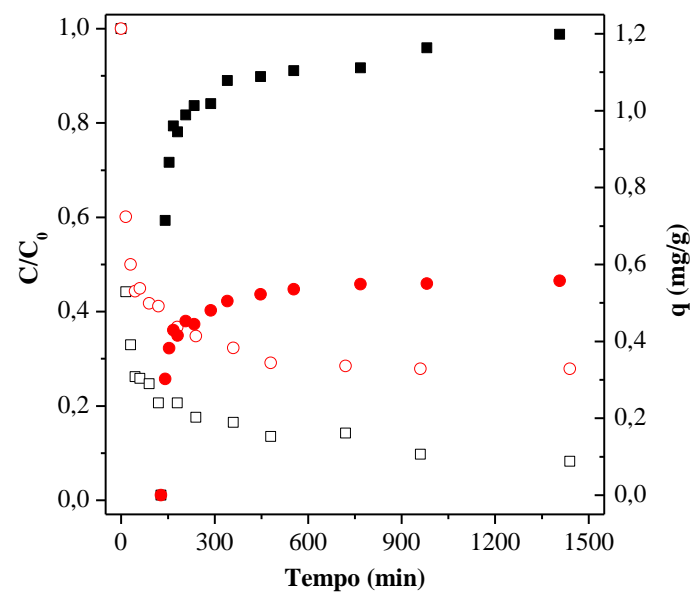

Tabela 1 - Parâmetros obtidos do modelo cinético de pseudo-primeira ordem e pseudosegunda ordem para a remoção dos íons metálicos Fe e Mn pelo bagaço de malte.

\begin{tabular}{|c|c|c|c|c|c|c|c|}
\hline & \multirow[b]{2}{*}{$q_{\exp }(\mathrm{mg} / \mathrm{g})$} & \multicolumn{3}{|c|}{ Pseudo-primeira ordem } & \multicolumn{3}{|c|}{ Pseudo-segunda ordem } \\
\hline & & $k_{1}$ (g/mg.h) & $q_{1}(\mathrm{mg} / \mathrm{g})$ & $\mathbf{R}^{2}$ & $k_{2}$ (g/mg.h) & $\mathbf{q}_{2} \quad(\mathrm{mg} / \mathrm{g})$ & $\mathbf{R}^{2}$ \\
\hline Ferro & 1,1 & 0,1473 & 0,6317 & 0,7329 & 2,1870 & 1,1957 & 0,9989 \\
\hline Manganês & 0,5 & 0,2331 & 0,4879 & 0,8810 & 4,6872 & 0,5643 & 0,9998 \\
\hline
\end{tabular}

\section{CONCLUSÃO}

Por meio dos resultados da caracterização da qualidade das águas subterrâneas foi possível estabelecer os íons $\mathrm{Fe}^{2+}$ e $\mathrm{Mn}^{2+}$ como poluentes-alvo no estudo da biossorção. $\mathrm{O}$ bagaço de malte utilizado no processo de biossorção apresentou uma capacidade máxima de remoção de $1,1 \mathrm{mg} / \mathrm{L}$ e $0,5 \mathrm{mg} / \mathrm{L}$, respectivamente para Fe e $\mathrm{Mn}$, com uma concentração de biossorvente de 2,0 g/L. O modelo cinético de pseudo-segunda teve melhor ajuste para os dados experimentais cinéticos, apresentando coeficientes de correlação linear próximo a 1 . $\mathrm{O}$ estudo cinético também permitiu verificar que há uma alta velocidade de adsorção no início 
do processo. De forma geral, conclui-se que o bagaço de malte é um biossorvente potencial para a remoção de metais em soluções aquosas.

\section{REFERÊNCIAS}

AQUARONE, E.; BORZANI, W.; SCHMIDELL, W., LIMA, U de A. Biotecnologia Industrial: Biotecnologia na produção de alimentos. Editora Blücher, São Paulo, 2001.

AKCIL, A.; KOLDAS, S. Acid Mine Drainage (AMD): causes, treatment and case studies. $J$. Clean. Prod., v. 14, p. 1139-1145, 2006.

APHA/AWWA/WEF. Standard Methods for the Examination of Water and Wastewater, 22 Ed. American Public Health Association, Washington, 2012.

BRASIL. Ministério da Saúde - Portaria 2.914 de 12 de dezembro de 2011.

FADEL, M.; HASSANEIN, N. M.; ELSHAFEI, M. M.; MOSTAFA, A. H.; AHMED, M. A.; KHATER, H. M. Biosorption of manganese from groundwater by biomass of Saccharomyces cerevisiae, HBRC Journal (In press).

FERRAZ, A.I.; AMORIM, C.; TAVARES, T.; TEIXEIRA, J. A. Chromium(III) biosorption onto spent grains residual from brewing industry: equilibrium, kinetics and column studies. Int. J. Environ. Sci. Technol., v. 12, p. 1591-1602, 2015.

FONTANA, K.B.; CHAVES, E.S.; SANCHEZ, J.D.; WATANABE, E.R.; PIETROBELLI, J.M.; LENZI, G.G. Textile dye removal from aqueous solutions by malt bagasse: Isotherm, kinetic and thermodynamic studies. Ecotoxicol. Environ. Saf., v. 124, p. 329336, 2016.

IBRAHIM, W. M. Biosorption of heavy metal ions from aqueous solution by red macroalgae. J. Hazard. Mater., v. 192, p. 1827-1835, 2011.

IGWE, J.C.; ABIA, A. A. Adsorption isotherm studies of $\mathrm{Cd}$ (II), Pb (II) and $\mathrm{Zn}$ (II) ions bioremediation from aqueous solution using unmodified and EDTA-modified maize cob. Eclet. Quim., v. 32, p. 33-42. 2007.

JI, G.L.; LI, H.Y. Electrotatic adsorption of cations. In: YU, T. R. Chemistry of variable charge soils. Oxford University Press, New York, 1997.

LAGERGREN, S. Zur theorie der sogenannten adsorption gelöster stoffe. Handlinger, v. 24, p. $1-39,1898$.

SANTOS, M. S.; RIBEIRO, F. M. Cervejas e Refrigerantes. CETESB, São Paulo, 2012.

SANTOS, D. M. dos; BUKZEM, A. L.; ASCHERI, D. P. R.; SIGNINI, R.; AQUINO, G. L. B. de. Microwave-assisted carboxymethylation of cellulose extracted from brewer's spent grain. Carbohydr. Polym., v. 131, p. 125-133, 2015.

WANG, J.; CHEN, C. Biosorption of heavy metals by Saccharomyces cerevisiae: A review. Biotechnol. Adv., v. 24, p. 427-451, 2006.

ZHANG, Y.; ZHAO, J.; JIANG, Z.; SHAN, D.; LU, Y. Biosorption of Fe (II) and Mn (II) Ions from Aqueous Solution by Rice Husk Ash. Biomed. Res. Int., v. 2014, p. 1-10, 2014. 